\title{
Análisis de la Validez de Contenido de un Cuestionario de Evaluación del Aprendizaje Basado en Problemas. Un Enfoque Cualitativo
}

\author{
Carme Carrion ${ }^{(1,2) *}$, Mònica Soler ${ }^{(3)}$ y Marta Aymerich ${ }^{(1,4)}$ \\ (1) TransLab Research Group, Universidad de Girona, Emili Grahit 71, 17071 Girona, Cataluña-España. \\ (e-mail: carme.carrion@udg.edu) \\ (2) Agència de Qualitat i Avaluació sanitàries de Catalunya. Roc Boronat 85, 08005 Barcelona-España. \\ (3) Unidad de Educación Médica, Universidad de Girona. Emili Grahit 71, 17071 Girona, Cataluña-España. \\ (4) Universitat Oberta de Catalunya, Av Tibidabo 39-43, 08035 Barcelona, Cataluña-España.
}

${ }^{*}$ Autor a quien debe ser dirigida la correspondencia

Recibido Ago. 7, 2014; Aceptado Ago. 22, 2014; Versión final recibida Nov. 5, 2014

\begin{abstract}
Resumen
El objetivo de este estudio es analizar la validez de contenido de un cuestionario de evaluación de los valores y actitudes profesionales durante las sesiones de Aprendizaje Basado en Problemas (ABP). Este método se ha consolidado en las ciencias médicas que la Facultad de Medicina de la Universidad de Girona (España) implementa en su plan de estudios. Se ha contado con la colaboración de siete expertos que han respondido a preguntas abiertas. El análisis cualitativo de las opiniones de los expertos ha revelado cuáles son los ítems considerados relevantes en la evaluación. El cuestionario analizado es un instrumento válido para evaluar los valores y actitudes profesionales que se trabajan en las sesiones de ABP. Catorce de los veinte ítems son relevantes según la opinión de los expertos. Sin embargo, para mejorar la validez de contenido se deberían añadir tres nuevos ítems: autonomía del estudiante, uso apropiado del lenguaje no verbal y capacidad para controlar las emociones.
\end{abstract}

Palabras clave: aprendizaje basado en problemas, valores y actitudes profesionales.

\section{Analyzing the Validity of the Evaluation Questionnaire Content of Problem-Based Learning. A Qualitative Approach}

\begin{abstract}
The aim of this study is to analyze the validity of the content included in a standardized questionnaire used to evaluate components related to professional values and attitudes during Problem-Based Learning (PBL) sessions. PBL is a well-established learning method used in the medical sciences of the School of Medicine of the University of Girona (Spain) and is currently included in its curriculum. The validity of the content of the instrument was determined by posing open-ended questions to seven experts. A qualitative analysis of the experts' opinions revealed the items that are relevant for evaluating the professional training of medical students. The questionnaire appears to be a valid tool for evaluating the professional values analyzed during PBL sessions. Fourteen of the twenty items are relevant. To improve the content validity of the questionnaire, three new items should be added: student autonomy, using appropriate nonverbal language, and capacity of controlling emotions.
\end{abstract}

Keywords: problem-based learning, professional values and attitudes 


\section{INTRODUCCIÓN}

Las Facultades de Medicina tienen como objetivo que los médicos que se gradúen sean capaces de servir a la comunidad y de aportar avances en el campo de la medicina (Al Alwan et al., 2011). La formación debería preparar a los estudiantes de medicina para que sepan gestionar los problemas con los que se encontrarán en el futuro y ofrecerles las competencias necesarias para que sean estudiantes autodidactas en lugar de destinatarios pasivos de la información (Dolmans et al. 2006). Un plan de estudios bien diseñado debería garantizar que los estudiantes adquiriesen estas competencias. Dentro de la disciplina de la formación médica, el Aprendizaje Basado en Problemas (ABP) es un enfoque curricular que se basa en la educación centrada en el estudiante (Lermanda, 2007; Branda, 2009). Aunque utilizado en otras áreas distintas a las ciencias de la salud, como por ejemplo la ingeniería (Fernández y Duarte, 2013; Vega et al., 2014), la ventaja pedagógica del ABP es la capacidad visible que ofrece de potenciar, mediante estos procesos de aprendizaje, unas habilidades óptimas en razonamiento clínico y el desarrollo tanto de una base de conocimiento flexible como de competencias para la formación autodidáctica, necesarias para seguir formándose a lo largo de la vida (Kelson y Distlehorst, 2000). El enfoque del ABP incluye cuatro condiciones cruciales en el proceso de aprendizaje: una base de conocimientos bien estructurada, un aprendizaje activo, una interacción colaborativa del estudiante y un contexto diseñado para promover la motivación personal gracias al establecimiento de metas pragmáticas (Margetson, 1994). El ABP es un enfoque educativo que fomenta que los estudiantes "aprendan a aprender" y que trabajen de manera cooperativa en grupos para buscar soluciones (Haghparast et al., 2007).

El ABP es la estrategia de aprendizaje básica que se utiliza en el plan de estudios de Medicina de la Universidad de Girona y significa más del $60 \%$ de cada módulo educativo. Se centra no sólo en la evaluación de los conocimientos y las competencias médicas, sino también en los valores y en las actitudes profesionales. Estos valores y actitudes, sintetizados en una sola palabra como profesionalismo, son esenciales en la práctica médica y, por lo tanto, deberían formar parte de la formación de los estudiantes de medicina. El profesionalismo tiene un impacto en la atención que se ofrece al paciente, en los resultados de salud, en las relaciones terapéuticas y en la percepción y confianza de los pacientes en la atención sanitaria y en sus profesionales (Cohen, 2006; Hammer et al., 2003; Maastricht University, 2010).

Existen escasos antecedentes del proceso a seguir en la evaluación del profesionalismo, especialmente en un contexto de ABP en las ciencias de la salud. Asimismo, la investigación en educación médica se encuentra hoy por hoy en una etapa inicial, sobretodo si lo comparamos con otras áreas de la investigación biomédica. En su mayor parte, la investigación en educación médica se limita a una descripción de casos o comentarios sobre experiencias con mayor o menor difusión (Centeno y Martinez-Carretero, 2003). En los últimos años se ha incrementado el interés por el estudio de las metodologías y su repercusión en el profesionalismo de los estudiantes. Así, en la Facultad de Medicina de Durham, en el Reino Unido se ha llevado a cabo la evaluación de las propiedades métricas de un test sobre profesionalismo, mediante preguntas de respuesta seleccionada (Tiffin et al., 2011). En este estudio se pudo apreciar que la herramienta diseñada no era suficientemente eficaz ni fiable y puso de manifiesto la necesidad de desarrollar nuevas estrategias para poder evaluar el profesionalismo en los estudiantes de medicina. Parece necesario analizar bien los avances en profesionalismo de los futuros médicos y para ello conviene disponer de herramientas útiles, que sean eficaces y válidas. Varios autores (O'Sullivan y Toohey, 2008; Swick, 2000) sugieren que los estudiantes con un buen profesionalismo cometerían menos errores médicos y, en caso de producirse, podrían disponer de valores personales y recursos suficientes que les ayudaran a afrontar los errores cometidos de forma más honesta y eficaz.

Hoy por hoy no existe un consenso en educación médica sobre como valorar el profesionalismo entre los estudiantes de grado. Una revisión bibliográfica (Wilkinson et al., 2009) puso de manifiesto que el profesionalismo puede ser evaluado de distintas formas que van desde la observación durante el encuentro clínico a la opinión de los pacientes, pasando por los pacientes simulados, entre otras. Se han descrito métodos útiles para los estudios de postgrado (Sánchez et al., 2014; Aburto et al, 2012) o como máximo para los cursos clínicos de grado (Sutton et al., 2007). El reto se encuentra pues en poder evaluar el profesionalismo de los primeros cursos de medicina. El ABP proporciona el escenario necesario para poderlo llevar a cabo sin necesidad de desarrollar exámenes específicos o esperar a que los estudiantes estén en contacto con pacientes. Existen modelos de cuestionarios sobre profesionalismo que se han aplicado en el ABP (Elizondo-Montemayor, 2007) pero no parece que hayan sido evaluados en cuando a su validez y/o fiabilidad.

En la Facultad de Medicina de la Universidad de Girona se utiliza un cuestionario (o rúbrica) para evaluar los valores y las actitudes profesionales de los estudiantes desde el primer curso. Por lo tanto, representa una oportunidad para aportar conocimiento en el área de educación médica investigando sobre las propiedades métricas del instrumento. Previamente se evaluó tanto la fiabilidad en términos consistencia interna (Aymerich 
et al., 2011), como la correlación de las medidas del cuestionario con los aprendizajes de contenido curricular (Carrion et al., 2013). El objetivo de este estudio es analizar la validez de contenido del instrumento para evaluar los valores y las actitudes profesionales en las sesiones de ABP.

\section{MATERIALES Y MÉTODOS}

\section{Contexto}

La Facultad de Medicina de la Universidad de Girona empezó a impartir estudios de grado en 2008 y desde el primer momento la estrategia educativa escogida fue el ABP. Los estudiantes trabajan durante los 6 años que duran los estudios en pequeños grupos autodirigidos (en las sesiones de ABP) para definir, llevar a cabo y reflexionar sobre una tarea de "investigación", que a menudo hace referencia a un problema de algún caso real. El tutor actúa como facilitador. Los estudiantes se dividen en grupos de 10 y se reúnen con el tutor facilitador tres veces por semana para discutir sobre un caso. Durante la primera sesión, de dos horas, los elementos de aprendizaje se van generando a partir del conocimiento de los estudiantes, y en la segunda y tercera sesión, también de dos horas cada una, los estudiantes realizan una puesta en común y contrastan lo aprendido. Las competencias genéricas en las sesiones de ABP se evalúan mediante un cuestionario estandarizado que se describe a continuación y que se implementa sistemáticamente en todos los módulos. Significa por lo menos el $40 \%$ de la nota final de cada módulo. La implementación incluye una autoevaluación, una evaluación conjunta de los compañeros del grupo y una evaluación del tutor.

\section{Instrumento}

El cuestionario estandarizado para evaluar el desempeño de los estudiantes en las sesiones de ABP está basado en uno previo (Vecchi et al., 2004) a partir del cual uno de los autores (Branda, 2009) hizo una posterior adaptación del mismo siendo profesor visitante en la Facultad de Medicina de la Universidad de Girona. El autoaprendizaje en el que se basa el ABP ayuda a desarrollar habilidades y competencias ligadas al profesionalismo. En consecuencia, el cuestionario contiene veinte ítems relacionados con diferentes valores y actitudes, y están divididos en cuatro ámbitos: habilidades de aprendizaje, comunicación, responsabilidad y relaciones interpersonales.

La dimensión de las habilidades de aprendizaje implica la adquisición de estrategias y competencias de razonamiento que pueden estar relacionadas con conocimientos nuevos o previos. En esta dimensión se incluyen los cinco criterios siguientes: 1) habilidad para identificar áreas de aprendizaje relevantes a los problemas; 2) utilización del conocimiento previo para identificar áreas de aprendizaje relacionadas con los problemas; 3) contribución a la preparación del plan de trabajo del grupo, escogiendo las áreas de aprendizaje y justificando de forma razonada; 4) grado de comprensión de las áreas de conocimiento o materias identificadas explicándolas y sintetizándolas razonadamente; y 5) realización de un análisis crítico, contrastando y aplicando los conocimientos aportados al problema.

La dimensión de comunicación hace referencia a los siguientes aspectos: 1) expresión con claridad y precisión evitando las redundancias; 2) capacidad de hacer comentarios aclaratorios respecto las discusiones producidas en el grupo; 3) grado de participación en las discusiones escuchando de forma activa, teniendo en cuenta si se interrumpe o no a los compañeros; 4) frecuencia de emisión de comentarios relevantes respecto a las discusiones del grupo; y 5) realización de síntesis claras de la información discutida.

La dimensión de responsabilidad incluye varios criterios relacionados con el estudiante individualmente. A saber: 1) la asistencia a las sesiones de ABP, frecuencia de la inasistencia y, en tal caso, si se ha justificado de forma razonada; 2) la puntualidad a las sesiones, frecuencia de la no puntualidad y, en tal caso, si se ha justificado de forma razonada; 3) frecuencia con la que se cumple con la responsabilidad de llevar a cabo la búsqueda de información; 4) realización de acciones de mejora para corregir las carencias o debilidades; y 5) grado de contribución al aprendizaje del grupo aportando nuevos conocimientos.

Finalmente, la dimensión de relaciones interpersonales implica los cinco criterios que se enumeran a continuación: 1) contribución a ordenar una discusión teniendo en cuenta lo que han expresado los compañeros; 2) expresión con un lenguaje adecuado, siendo respetuoso con sus compañeros; 3) realización de críticas de manera constructiva; 4) grado de comportamiento cooperativo dirigiendo las acciones y comentarios al trabajo del grupo y no a los intereses individuales; y 5) grado de colaboración con los compañeros ayudándoles a la mejora de su aprendizaje. Para obtener la puntuación final de la evaluación, a cada ítem se le puede dar 0,1 o 2 puntos en función de hasta qué punto se ha alcanzado el objetivo, siendo la puntuación máxima 40 puntos.

\section{Análisis de la validez de contenido}


La validez de un instrumento hace referencia a la capacidad que tiene de medir y describir lo que se supone que debe medir y describir. En concreto, la validez de contenido tiene como objetivo comprobar el grado en que un instrumento refleja un dominio específico de contenido de lo que se mide, es decir, el grado en que la medición representa al concepto medido. En este estudio se trató de averiguar si el contenido (las preguntas) de la rúbrica o del cuestionario está basado en una opinión aceptada en general sobre valores y actitudes profesionales. La validez de contenido suele evaluarse a través de un panel o un juicio de expertos, que se define como una opinión informada de personas con trayectoria en el tema, que son reconocidas por otros como expertos cualificados en éste, y que pueden dar información, evidencia, juicios y valoraciones (EscobarPérez y Cuervo-Martínez, 2008). La validez de contenido en el presente estudio se estableció en la medida que los profesionales o expertos determinaban un contenido para las preguntas o aseveraciones que debería tener el instrumento y, en consecuencia, qué tan adecuado era el instrumento en relación con su juicio.

\section{Implicación de expertos y entrevistas}

Se eligieron siete expertos diferentes teniendo en cuenta tanto su pericia como docentes en el campo de la medicina como su experiencia clínica. Los expertos escogidos son profesionales de la medicina de reconocido prestigio internacional, especializados en la docencia médica de grado y postgrado que ejercen o bien han ejercido en centros universitarios distintos al que se ha desarrollado el instrumento. A continuación se describen brevemente las características de cada uno de ellos.

Experto 1. Jefe de servicio de medicina interna de un hospital universitario. Catedrático de universidad, habiendo sido decano de una facultad de medicina así como presidente de un colegio de médicos.

Experto 2. Catedrático de universidad en el área de ciencias básicas, habiendo sido decano de una facultad de medicina y presidente de diversas sociedades científicas de educación médica. Coautor de un libro blanco sobre la formación de médicos.

Experto 3. Catedrático de universidad en un hospital universitario de prestigio mundial habiendo estado implicado en la formación de residentes durante más de 30 años. Editor fundador de un libro de referencia para médicos internistas en Estados Unidos.

Experto 4. Epidemiólogo con larga experiencia en el campo de la gestión del conocimiento en ciencias de la salud. Profesor de universidad en el área de salud pública. Alta responsabilidad en sociedades científicas de bioética y en asociaciones de pacientes, siéndolo él mismo de larga duración.

Experto 5. Catedrático de patología médica en universidades de prestigio. Médico en diversos hospitales universitarios europeos. Editor fundador de un libro de referencia para médicos internistas en España.

Experto 6. Jefe de una unidad de educación médica de una facultad de medicina. Miembro del Consejo de diversas sociedades y revistas científicas de educación médica.

Experto 7. Catedrático de universidad en el área de ciencias básicas. Alta responsabilidad en sociedades científicas europeas de educación médica.

A todos ellos se les enviaron por correo electrónico preguntas abiertas individuales a las que respondieron durante el período de tiempo de junio-septiembre de 2011. Las preguntas que se formularon a los expertos fueron las siguientes: ¿Cuáles cree usted que deberían ser los valores y actitudes de un profesional de la medicina actualmente? ¿Es usted partidario de la formación y evaluación de estos valores y actitudes durante los estudios de grado? ¿Por qué? ¿Considera importante que la comunicación sea un aspecto relevante y a la vez sea evaluado durante los estudios de medicina? ¿Considera que es relevante trabajar aspectos relacionados con la responsabilidad durante los estudios de medicina? ¿Considera importante incluir aspectos relacionados con las relaciones personales? ¿Cuáles serían todos estos aspectos?

\section{Análisis cualitativo de los datos}

Los datos obtenidos se transcribieron literalmente una vez recibidas las respuestas de cada experto y se analizaron mediante un análisis cualitativo del contenido. Tres investigadores codificaron por separado los datos y luego compararon los resultados. Los investigadores llevaron a cabo el análisis mediante la codificación en nodos o categorías, equivalentes a las dimensiones del cuestionario y, en subnodos o subcategorías, equivalentes a los ítems dentro de cada dimensión, a fin de relacionar las respuestas de los expertos con las categorías definidas y dar visibilidad a las aportaciones de éstos. Ello permitió percibir por un lado los elementos conceptualmente coincidentes y, por otro, elementos de mejora no incluidos en el 
cuestionario. El análisis de los datos fue realizado primero de manera individual e independiente por parte de los tres investigadores y después se llevó a cabo un consenso mediante una puesta en común. En caso de desacuerdo, se abrió un debate entre los tres investigadores para alcanzar un acuerdo sobre la codificación. Este proceso conllevó recategorizar y renombrar parte del material para elaborar las categorías finales.

\section{RESULTADOS}

El análisis cualitativo de las opiniones de los expertos reveló cuáles son los ítems considerados relevantes en la evaluación de la formación profesional de estudiantes de medicina y que forman parte del cuestionario, y cuáles son los ítems que, a pesar de que algunos expertos los consideren importantes, no están incorporados en el instrumento (Tabla 1).

Tabla 1: Palabras clave identificadas por expertos en relación con los ítems clasificados en las cuatro dimensiones actuales del cuestionario analizado. $\mathrm{Nm}$ : no mencionado

\begin{tabular}{|c|c|c|c|}
\hline Habilidades de aprendizaje & Comunicación & Responsabilidad & Relaciones interpersonales \\
\hline $\begin{array}{l}\text { Identificación áreas de } \\
\text { aprendizaje }\end{array}$ & Expresión clara y precisa & Asistencia a las sesiones & $\begin{array}{l}\text { Contribución a ordenar la } \\
\text { discusión }\end{array}$ \\
\hline $\begin{array}{l}\text { Metodología de } \\
\text { autoaprendizaje }\end{array}$ & Información comprensible & Asistencia & Liderazgo \\
\hline Adquisición de conocimientos & Necesidades de comunicación & & Altruismo \\
\hline Adquisición de competencias & Fluidez & Puntualidad & \\
\hline $\begin{array}{l}\text { Reflexión sobre situaciones } \\
\text { específicas }\end{array}$ & Saber cómo expresarse & Puntualidad & Respeto \\
\hline Uso de conocimiento previo & Contribución a la claridad & $\begin{array}{l}\text { Búsqueda de las áreas de } \\
\text { conocimiento }\end{array}$ & No tener perjuicios \\
\hline \multirow[t]{2}{*}{$\begin{array}{l}\text { Conocimiento científico } \\
\text { suficiente }\end{array}$} & $\mathrm{Nm}$ & Utilización de tiempo & $\begin{array}{l}\text { Valoración de las ideas de los } \\
\text { demás }\end{array}$ \\
\hline & & $\begin{array}{l}\text { Responsabilidad individual y } \\
\text { social }\end{array}$ & Tacto \\
\hline $\begin{array}{l}\text { Preparación de un plan de } \\
\text { trabajo }\end{array}$ & Escucha activa & $\begin{array}{l}\text { Hacia la profesión, la sociedad } \\
\text { y uno mismo }\end{array}$ & $\begin{array}{l}\text { Respeto por autonomía y } \\
\text { dignidad del paciente }\end{array}$ \\
\hline \multirow[t]{2}{*}{$\mathrm{Nm}$} & Aprender a escuchar & Servicio a los ciudadanos & Respeto de las diferencias \\
\hline & Comunicación entre colegas & Responsabilidad social & \\
\hline $\begin{array}{l}\text { Comprensión de las áreas de } \\
\text { conocimiento }\end{array}$ & $\begin{array}{l}\text { Identificación de elementos de } \\
\text { comunicación }\end{array}$ & & Críticas constructivas \\
\hline \multirow[t]{2}{*}{$\mathrm{Nm}$} & & Corrección de debilidades & $\mathrm{Nm}$ \\
\hline & Comentarios relevantes & Esfuerzo & \\
\hline Análisis crítico & $\mathrm{Nm}$ & Honestidad & Comportamiento cooperativo \\
\hline Análisis crítico & & Aceptación de críticas externas & Adaptabilidad \\
\hline Búsqueda de la verdad & Síntesis de la información & Mejorará la competencia & Compañerismo \\
\hline Ser crítico & Puesta en común & & Flexibilidad \\
\hline \multirow[t]{10}{*}{ Habilidad de analizar } & $\begin{array}{l}\text { Habilidades comunicativas } \\
\text { para enseñar }\end{array}$ & $\begin{array}{l}\text { Contribución al aprendizaje del } \\
\text { grupo }\end{array}$ & \\
\hline & & $\mathrm{Nm}$ & Comportamiento colaborativo \\
\hline & & & Solidaridad \\
\hline & & & Empatía \\
\hline & & & $\begin{array}{l}\text { Negociación entre adultos y } \\
\text { autonomía }\end{array}$ \\
\hline & & & Empático \\
\hline & & & Altruismo \\
\hline & & & Disponibilidad \\
\hline & & & Actitud compasiva \\
\hline & & & Altruismo \\
\hline Otras sugerencias & Otras sugerencias & Otras sugerencias & Otras sugerencias \\
\hline Autonomía & Comunicación doctor-paciente & $\begin{array}{l}\text { Capacidad de hacer } \\
\text { seguimiento }\end{array}$ & $\begin{array}{l}\text { Relaciones emocionales } \\
\text { saludables }\end{array}$ \\
\hline Razonamiento clínico & Lenguaje no verbal apropiado & & \\
\hline Ser independiente & & & \\
\hline
\end{tabular}


seis de ellos no son mencionados por los expertos y, por lo tanto, no son considerados relevantes, mientras que los otros catorce sí que son mencionados en repetidas ocasiones.

En la dimensión "habilidades de aprendizaje", los ítems preparación de un plan de trabajo, contribución al aprendizaje del grupo y comprensión de las áreas de conocimiento no son mencionados por ninguno de los expertos consultados. En cambio, aspectos como la autonomía, ser independiente o el razonamiento clínico son elementos mencionados por dos de ellos, pero no forman parte del cuestionario. En la dimensión "comunicación", los aspectos que los expertos no mencionan son la contribución de los estudiantes a la claridad de los conceptos estudiados y la capacidad de realizar comentarios relevantes. Por otro lado, de los comentarios de los expertos se pueden extraer nuevas sugerencias. Se considera importante incluir un ítem sobre lenguaje no verbal apropiado, y que la comunicación entre el médico y el paciente es un aspecto importante. Las competencias comunicativas son muy importantes para cinco de los siete expertos consultados. La comunicación se entiende en dos planos diferentes: entre colegas profesionales y con los pacientes. Este último aspecto no se evalúa en las sesiones de ABP.

Por lo que se refiere a la dimensión "responsabilidad", uno de los expertos incluye como relevante el ítem capacidad de hacer el seguimiento de un caso a lo largo del tiempo. Los ítems relacionados con la puntualidad y la asistencia a las sesiones sólo los menciona uno de los expertos. La mayor parte de los expertos consultados consideran importante la responsabilidad, tanto social como individual, y ésta última entendida como el hecho de que los estudiantes lleven a cabo investigaciones sobre campos específicos de conocimiento. Finalmente, los ítems de la dimensión "relaciones interpersonales" son los más mencionados por parte de los expertos. Ninguno de los expertos menciona el ítem de crítica constructiva, pero una vez más proponen un nuevo ítem; en este caso relacionado con el control de las emociones. Tres expertos diferentes consideran que la empatía es una de las actitudes más importantes.

\section{DISCUSIÓN}

El cuestionario analizado es un instrumento efectivo para llevar a cabo la evaluación del profesionalismo en estudiantes de medicina. Este estudio permite optimizar este instrumento de evaluación en términos de validez de contenido. Al comparar el cuestionario con la opinión de los expertos, se puede afirmar que éste incorpora la mayor parte de los aspectos que los expertos profesionales consideran importantes, exceptuando la autonomía del estudiante, un lenguaje no verbal apropiado y el control de las emociones. Se debería considerar la opción de incorporar estos nuevos elementos al cuestionario, ya que se consideran elementos importantes para desarrollar unas buenas competencias médicas profesionales. Por otro lado, los expertos no consideran relevantes algunos ítems del cuestionario, como la participación de los estudiantes en la preparación del plan de trabajo, su habilidad para realizar comentarios relevantes, su contribución a la claridad de los conceptos estudiados, su capacidad para realizar críticas constructivas, su contribución al aprendizaje del grupo y su comprensión de las áreas de conocimiento o materias. Debería llevarse a cabo más investigación para determinar si estos seis aspectos son necesarios o no.

El profesionalismo y la conducta ética son elementos esenciales en la práctica médica. Por este motivo, no sólo se debe dar importancia a la formación y a la evaluación de los conocimientos médicos, sino también a los valores profesionales. Hasta hace poco se pensaba que estos valores y el razonamiento crítico se conseguían de manera espontánea. Promover el profesionalismo es una parte muy importante de la formación médica e incluye un conjunto de valores, actitudes y aspectos relacionales que no son ni fijos ni homogéneos. O'Sullivan y Toohey (2008) analizaron el desarrollo profesional de estudiantes de medicina y concluyeron que para que puedan adquirir valores y actitudes profesionales relevantes, su formación debe incluir aspectos específicos del profesionalismo, así como valores éticos y humanísticos, como por ejemplo la integridad y la honestidad. Aspectos como la empatía, la responsabilidad, la honestidad y el respeto se consideran elementos de profesionalismo médico muy importantes. En la actualidad no existe una definición clara ni un consenso sobre lo que conlleva el profesionalismo y es necesario disponer de esta definición para poder medirla. Sin embargo, Wilkinson et al., (2009) establecieron cinco dimensiones para medir el profesionalismo: adhesión a unos principios de práctica ética, interacciones efectivas con los pacientes, interacciones efectivas con otros profesionales de la salud, confiabilidad y compromiso con la competencia. Rosselot (2006) también estableció un listado de las diferentes actitudes que se deben tener en cuenta en los estudios de medicina basándose en los principios y en las competencias que pueden definir lo que es el profesionalismo. Este listado incluye aspectos como la honestidad, la integridad, la empatía, la confianza y el compromiso, entre otros. A pesar de que sea ampliamente sabido y aceptado que el sistema del ABP ayuda a desarrollar valores profesionales (Kelson y Distlehorst, 2000), dichos valores también deben evaluarse.

En la Facultad de Medicina de la Universidad de Girona las sesiones de ABP representan herramientas clave para el aprendizaje de valores profesionales y para poder ponerlos en práctica. El Espacio Europeo de Educación Superior sugiere que debe realizarse un cambio global en los procesos de evaluación para poder 
considerar las competencias transversales y globales con mayor profundidad. Por este motivo, parece importante evaluar la adquisición de valores profesionales durante las sesiones de ABP. Cuando se evalúa el profesionalismo, tanto los tutores como los estudiantes ponen más énfasis en la importancia de este aspecto y de ahí la necesidad de que exista una herramienta efectiva para evaluar los valores profesionales.

Los valores profesionales relacionados con las habilidades de aprendizaje, la comunicación, la responsabilidad y las relaciones interpersonales siempre están presentes en las sesiones de ABP y evaluarlos facilita que los estudiantes sean más conscientes de su importancia. El cuestionario es una herramienta útil para que se establezcan criterios estandarizados entre los diferentes tutores y facilita la sistematización de la evaluación. La autoevaluación, la evaluación de los compañeros y la evaluación del tutor ayudan a los estudiantes a tomar consciencia de su propio desarrollo en el momento de adquirir habilidades profesionales. De todas formas, el éxito del proceso de evaluación también depende de otros aspectos, como por ejemplo de cómo se implementa.

Finalmente, el cuestionario de evaluación del ABP evalúa la adquisición de competencias profesionales en entornos pequeños y controlados, como es el caso de los grupos de trabajo. Aunque los grupos varíen en función del módulo, esta evaluación no puede desmostar las actitudes de los estudiantes en la vida profesional real. Asimismo, también puede ocurrir que los estudiantes asuman determinados roles de manera automática y rutinaria, pero que no los interioricen totalmente. Sería interesante implementar otro cuestionario más orientado hacia aspectos clínicos para evaluar las habilidades profesionales cuando los estudiantes empiecen a trabajar con pacientes u otros profesionales de la salud. Algunas de las propuestas realizadas por parte de los expertos consultados van en esta línea y encajarían perfectamente con un cuestionario de este tipo.

Existen ciertas limitaciones en este estudio que se deben tener en cuenta. Se usaron diferentes idiomas para la extracción de datos de los comentarios de los expertos en función de su idioma materno (catalán, inglés y español) y los resultados se compararon con la versión en inglés del cuestionario que utilizamos en la Facultad de Medicina de la Universidad de Girona. A veces, no es sencillo encontrar palabras o expresiones que signifiquen exactamente lo mismo en el idioma original y en inglés. Los matices semánticos pueden ser difíciles de detectar y ello puede conllevar que algunos de los aspectos mencionados por los expertos no encajen completamente con los ítems reflejados en el cuestionario. Asimismo, los expertos provienen de diferentes contextos y culturas. Por este motivo, es difícil definir qué valores y actitudes dependen del contexto y, en consecuencia, pueden ser susceptibles de cambiar cuando se apliquen en otro contexto específico.

\section{CONCLUSIONES}

El instrumento analizado es válido para evaluar los valores profesionales que se trabajan en las sesiones de ABP. Según la opinión de los expertos, catorce de los veinte ítems incluidos en el cuestionario que diseñamos son relevantes y, por lo tanto, deben formar parte de una herramienta para evaluar las actitudes y los valores profesionales. Para mejorar la validez de contenido del instrumento se deberían añadir tres nuevos ítems: autonomía del estudiante, uso de un lenguaje no verbal apropiado y capacidad para controlar las emociones. Investigación ulterior debería confirmar si los seis ítems no considerados importantes por los expertos deben incluirse o no. También sería adecuado diseñar una nueva versión del cuestionario para evaluar, en los estudios universitarios de medicina, las actividades clínicas y no únicamente las actividades realizadas en las sesiones de ABP.

\section{REFERENCIAS}

Al Alwan I., M. Al-Moamary, N. Al-Attas, A. Al Kushi, E. AlBanyan, M. Zamakhshary, H.M.F. Al Kadri, H. Tamim, M. Magzoub, A. Hajeer y A. Schmidt, The Progress Test as a Diagnostic Tool for a New PBL Curriculum. Education for Health, 24 (3), 493 (2011).

Aburto, M. y M. Sánchez, Diseño de un cuestionario para identificar realidades de la práctica de la Medicina Basada en Evidencias en médicos residentes: Uso de redes semánticas modificadas. Revista de investigación en educación médica, 1, (4), 190-198 (2012)

Aymerich M., C. Carrion, M. Soler y L.A. Branda, Evaluation of medical students' professional values and attitudes. The Network TUFH International Conference, Graz, (2011).

Branda L. A., El aprendizaje basado en problemas: De herejía artificial a res popularis. Educ Med, 12, 11-23, (2009)

Carrion, C., M. Soler y M. Aymerich, Association of professional training evaluation to content evaluation. 3rd World Conference on Learning, Teaching and Educational Leadership - WCLTA 2012. Procedia - Social and Behavioral Sciences, 93, 1911-1915, (2013). 
Cohen, J., Professionalism in medical education, an American perspective: From evidence to accountability. Medical Education, 40, 607-617 (2006).

Centeno A.M. y J.M. Martínez-Carretero, Innovaciones, investigación y evidencias en educación médica. La colaboración EMBE (Educación Médica Basada en la mejor Evidencia). Educ Med, 6, 26-30 (2003).

Dolmans, D.H.J.M. y H.G. Schmidt, What do we know about cognitive and motivational effects of small group tutorials in problem-based learning? Adv Health Sci Educ, 11, 321-336 (2006).

Elizondo-Montemayor, L., Evaluación formativa y sumativa de la sesión tutorial de aprendizaje basado en problemas utilizando un sistema de rúbricas de referencia. Revista Avances, 4 (13), 51-59, (2007).

Escobar-Pérez, J. y A. Cuervo-Martínez, Validez de contenido y juicio de expertos: una aproximación a su utilización. Avances en Medición, 6, 27-36 (2008).

Fernández, F.H. y J.E. Duarte, El aprendizaje basado en problemas como estrategia para el desarrollo de competencias específicas en estudiantes de ingeniería. Form Univ, 6 (5), 29-38 (2013).

Haghparast, N., P.P. Sedghizadeh, C.F. Shuler, D. Ferati y C. Christersson, Evaluation of student and faculty perceptions of the PBL curriculum at two dental schools from a student perspective: a cross-sectional survey. European Journal of Dental Education, 11, 14-22 (2007)

Hammer, D., B. Berger, R. Beardsley y M. Easton, Student Professionalism. American Journal of Pharmaceutical Education, 67(3),1-29 (2003).

Kelson, A.C.M. y L.H. Distlehorst, Groups in problem-based learning (PBL): Essential elements in theory, \& practice. In: DH Evenson \& CE Hmelo (Eds), Problem-Based Learning: a Research Perspective on Learning Interactions (Mahwah, NJ, Lawrence Erlbaum Associates) (2000).

Lermanda S.C., Aprendizaje basado en problemas (ABP): una experiencia pedagógica en medicina. Rexe. Revista de Estudios y Experiencias en Educación, 11, 127-143 (2007)

Maastricht University, How to assess professionalism? [cited $2010 \quad 26$ February]; http://www.unimaas.nl/default.asp?template=werkveld.htm\&id=J555UERP57251SKJ3JG1\&taal=nl (2010).

Margetson, D., Current educational reform and the significance of problem-based learning. Stud Higher Edu, 19, $5-19$ (1994).

O'Sullivan A. y S.M. Toohey, Assessment of professionalism in undergraduate medical students. Med Teach, $30,280-286$ (2008).

Rosselot, E., Dimensions of medical professionalism. Perspectives for the twenty-first century. Rev Méd Chile, $134,657-664$ (2006).

Sánchez G. y F. Cisterna, La evaluación de los aprendizajes orientada al desarrollo de competencias en Odontología. Edu Méd Sup, 28(1), (2014).

Sutton, A. H., M.A. Chacalo, G. de la Cruz Flores, O.F.R. de la Roche, C.L. Montalvo y P.V. Puig, Construcción y validación de un instrumento para evaluar ambientes clínicos de aprendizaje en las especialidades médicas. Gaceta Médica de México, 149, 394-405 (2013).

Swick H.M., Toward a normative definition of medical professionalism. Acad Med, 75, 612-616 (2000).

Tiffin P.A., G.M. Finn y J.C. McLachlan, Evaluating professionalism in medical undergraduates using selected response questions: findings from an item response modelling study. BMC Med Educ, 11, 43 (2011).

Vecchi, C., M. Del Valle y M. García Diéguez, Evaluación de los Estudiantes. En: Gutiérrez RR (editor). Programa de Medicina para la U.N.S. Planificación de una Carrera de Medicina basada en nuevos paradigmas, 141-146. GS Impresiones, Güemes. Argentina (2004). 
Vega, F., E. Portillo, M. Cano y B. Navarrete, Experiencias de aprendizaje en ingeniería química: diseño, montaje y puesta en marcha de una unidad de destilación a escala laboratorio mediante el aprendizaje basado en problemas. Form Univ, 7(1), 13-22 (2014).

Wilkinson T.J., W.B. Wade y L.D. Knock, A blueprint to assess professionalism: results of a systematic review. Acad Med, 84, 551-558 (2009). 
